\title{
Die Kirchen sind FSK-müde
} von Wilbelm Bettecken

\begin{abstract}
Mitte Mai dieses Jahres haben die christlichen Kirchen in den USA die Mitarbeit in der Institution der Filmselbstkontrolle aufgekündigt. Auf äbnliche Entwicklungen in Deutschland weist der folgende Beitrag hin.
\end{abstract}

Nervosität und Optimismus gleichzeitig im Biebricher Schloß in Wiesbaden am Rhein. Nervosität deshalb, weil die von den Kirchen benannten Prüfer in der Freiwilligen Selbstkontrolle der Filmwirtschaft (FSK) immer weniger mit ihrer Enttäuschung zurückhalten, weil sie sich in der praktischen Prüfarbeit auf verlorenem Posten fühlen und sie unverblümt vom Austreten sprechen. Optimismus deswegen, weil die einflußreichen Vertreter der Filmwirtschaft nicht so recht daran glauben wollen, daß es den Kirchen ernst ist mit den Austrittsabsichten. Aber auf die Frage "Verlassen die Kirchen die FSK?" - gestellt von Dieter Krusche in der gleichnamigen Diskussionsrunde im Filmforum des Zweiten Deutschen Fernsehens (ZDF) - antwortete Dr. Gerd Albrecht, Köln, der neue Filmbeauftragte der Evangelischen Kirche, unmißverständlich mit "Ja“. Allerdings schränkte er ein: "Wenn sich in nächster Zeit nichts Entscheidendes ändert." Und sein katholischer Kollege, Direktor Wilhelm Schätzler, Köln, sprach unverhohlen seine Befürchtungen aus, daß es zu dieser Änderung wohl nicht kommen werde. Die Entwicklung der letzten Jahre spricht dieser Erwartung zuwider.

Zunächst kann es den Prüfern auf dem Kirchenplatz in der FSK nicht gleich sein, daß sie in der Offentlichen Meinung zu Komplizen der Filmwirtschaft gemacht werden. „Wir veröffentlichen ausschließlich von der FSK geprüftes und freigegebenes Werbematerial. Im übrigen weisen wir darauf hin, daß bei der Freigabe von Filmen und Werbung auch die Kirchen mitwirken." Diese beiden Sätze aus dem Antwortbrief der Chefredaktion einer großen Regionalzeitung auf die Beschwerde eines Lesers, er werde jeden Freitag mit einer Flut „Sex- und Crime-Propaganda“ in den Filmanzeigen überschüttet, erhellen schlaglichtartig die Situation der Kirchenprüfer. Sie werden von vielen Beobachtern als Renommierstücke für eine Institution angesehen, deren Image in den letzten Jahren sehr gelitten hat. Die undifferenzierte und daher schlicht unwahre Behauptung, die Kirchen seien für alle Freigabeentscheidungen mit-verantwortlich, wie sie in dem zitierten Brief enthalten ist, die vortäuschen will, es sei doch alles in Ordnung, kann aber von der tatsächlichen Situation der FSK nicht ablenken. Den Kirchenprüfern wird also eine Feigenblattfunktion zugeschoben, die schlechthin unerträglich ist. Wer nur ein wenig Einblick in die Arbeit der FSK hat, weiß, und wer bei der Betrachtung des heutigen Filmangebots nur ein wenig nachdenkt, ahnt, bei wievielen Filmen und bei noch mehr Werbematerial die Prüfer auf dem Kirchenstuhl ein schlichtes „Nein“ sagen müssen.

Als der honorige Repräsentant der deutschen Filmwirtschaft, Curt Oertel, in den Jahren 1948/1949 durch die deutschen Lande zog, um Freunde für den Gedanken einer Selbstkontrolle des Films in Westdeutschland zu werben, waren die Kirchen

Der Verfasser ist Leiter der Diözesanstelle für Film, Funk, Fernsehen, Presse und der Bischöflichen Pressestelle im Bistum Essen. Als Filmjournalist begleitet er die Arbeit der Freiwilligen Selbstkontrolle der Filmwirtschaft (FSK) seit Beginn ihrer Tätigkeit am 18.7.1949 kritisch. Seit April 1951 ist er Prüfer, zunächst benannt durch den Deutschen Bundesjugendring und seit 1962 durch die Katholische Kirche. 
gern zur Mitarbeit bereit. Aber kaum zwei Jahre nach Beginn der Arbeit, in der sogenannten „Sünderin “-Affäre, kam es erstmals zum Auszug der Kirchen aus der FSK. Dieser vielfach als unfreiwillige Propaganda-Aktion für einen schwachen Film mißdeutete "Skandal" hatte eine Umbildung der FSK zur Folge. Erst von diesem Augenblick an war die Parität zwischen Filmwirtschaft und Offentlicher Hand im Arbeitsausschuß hergestellt, und erst damit war das demokratische Selbstverwaltungsorgan geschaffen, das später auch für andere Wirtschaftszweige Vorbild wurde. Obwohl die Grundsätze der FSK seitdem bis Ende $1970 \mathrm{im}$ wesentlichen unverändert geblieben sind, kam es im Laufe der folgenden Jahre aufgrund von Erfahrungen aus der Prüfpraxis zu wichtigen Anderungen in Verfahrensfragen, immer mit dem Ziel, die Freigabeentscheidung zu verbessern.

Die Zahl der Zeitgenossen, die sich beim Blick auf die Filminserate der Freitagsausgaben der Tageszeitungen fragen, was sich die FSK wohl bei der Freigabe vieler Werbeelemente gedacht habe, oder gar, ob es überhaupt noch eine FSK gebe, ist in den letzten beiden Jahren erheblich gewachsen. Damit wird die Frage nach der Wirksamkeit einer solchen Kontrolle heute mit aller Deutlichkeit gestellt werden müssen. Eng damit verbunden ist auch die Frage nach dem Sinn einer kirchlichen Mitwirkung an weithin unbefriedigenden Entscheidungen.

Einer langen Aufzählung von Titeln bedarf es nicht, um zu verdeutlichen, wie fragwürdig die Grundsätze geworden sind. Dabei soll an „diskutable“ Filme - im echten Sinn des Wortes - gar nicht gedacht werden. Es genügen einige Beispiele, die auch im Sinne des Jugendschutzes bedeutsam sind: "Willst du ewig Jungfrau bleiben?", "Wenn im P... das Licht ausgeht" (wobei die Werbung selbstverständlich einen Weg findet, die fehlenden Pünktchen durch Buchstaben zu ersetzen), „Sex pervers“, „Geilermanns Töchter", „Stoßzeit“, „Im Rausch der Wollust“, „Hörig bis zur letzten Sünde“, „Ritter Orgas muß mal wieder", "Siegfried - das sagenhafte Liebesleben der Nibelungen“, „Frau Professor kann's noch besser “. Dazu die Titel, die von einer Brutalisierung des Sex zeugen: „Erotik in der Folterkammer,“ "Hexen bis aufs Blut gequält", „Der Todesschrei der Hexen“. Oder auch religiös relevante Titel, wie "Django spricht das Nachtgebet", "Sein Gebetbuch war der Colt", "Gott vergibt - Django nie“, „Django spricht kein Vaterunser“, "Requiem für Django", „Ein Halleluja für Django “, „Haß war sein Gebet“, „Andere beten, Django schießt", „Vier für ein Ave-Maria“, „Stoßgebet für drei Kanonen“, „Beichte einer Liebestollen“. Schließlich die Imperative der Gewalt: „Leg ihn um, Django“, „Töte, Ringo, töte“, „Töte alle und kehr allein zurück“, „Laßt uns töten, Companeros ${ }^{\alpha}$.

Entsprechende Beispiele aus der Werbung, die wie die Titel allen Kindern und Jugendlichen zugänglich ist, sind leicht zu finden. Nur ein Beispiel: „Sie lieben und leben wie Tiere in freier Wildnis, ohne Gesetz, ohne Moral, ohne Hemmungen “ ("Verboten “, mit FSK-Freigabe).

Auch Filme, die freigegeben sind, obwohl sie bei nur etwas konsequenter Handhabung der Grundsätze nicht oder wenigstens nicht in der entsprechenden Fassung hätten freigegeben werden dürfen, können in die Reihe der Beispiele eingereiht werden, die die Aufweichung der Grundsätze dokumentieren. Darunter finden sich vor allem jene schnell zusammengezimmerten Filmchen, die mit der Kennmarke "Porno“ Geschäfte machen wollen. Dazu gehören ebenfalls Filme, vor allem aus den Werkstätten der Nachfahren der alten Römer, die mit Grausamkeit und Brutalität ihren Produzenten Rendite einbringen sollen. Zu denken ist auch an Filme, die nicht 
nur "Sex and Crime" bringen, sondern die eine brutalisierte und damit unmensch. liche Sexualität zum Gegenstand des Kinovergnügens machen. Die FSK kann sidt: nicht einmal rühmen, auf dem Gebiet des Militarismus oder des Rassismus konsequent gewesen zu sein. Aber auch die zahllosen sogenannten Aufklärungsfilme, die in Filmwirtschaftskreisen mehrfach als Großtaten für die Gesellschaft gefeiert worden sind - weil sie Kasse machten und deshalb die "Goldene Leinwand" bekamen, gehören zum großen Teil zu den fragwürdigen Entscheidungen. Tatsächlich schaut ihnen die kommerzielle Spekulation durch nahezu jede Einstellung.

Zur rechten Beurteilung etwa der suggestiven Kolle-„Vorlesungen“ mit ihren Eskalationen der vermeintlich gebrochenen Tabus sollten auch einmal die Erfahrungen der Eheberaterinnen und -berater herangezogen werden. Diese Frauen und Männer sprechen von den „Kolle-Geschädigten ${ }^{*}$. Sie berichten aus ihren Sprechstunden, daß sich junge Mädchen plötzlich nicht mehr normal finden, weil sie noch nicht über die entsprechenden sexuellen Erfahrungen verfügen. Eheleute sind enttäuscht voneinander, weil sie Kolles Stellungsspiele nicht nachzuturnen vermögen. Ehefrauen fragen sich bestürzt, ob sie jemals die rechten Gefühle beim Orgasmus erlebt haben, und Männer bekommen Hemmungen, weil sie es mit den Sexprotzen der Leinwand nidt aufzunehmen vermögen. Dazu kommt, daß sie Kolles sexuelle Emanzipationsideologie gedanklich gar nicht nachvollziehen können. Sie sind nicht einmal in der Lage, den Tarnmantel lauterer Aufklärungsabsicht über einem reinen Kommerzprodukt zu lüften.

Angesichts dieser vor fünf Jahren noch kaum vorstellbaren Aufweichung der Spruchpraxis muß gefragt werden, was das denn für ein Wirtschaftszweig - mit kulturellem Hintergrund - ist, der sich im Sinne eines echten Fortschritts einmal von den Jahrmärkten und Rummelplätzen über die Café-Häuser in repräsentative Lichtspieltheater hinaufentwickelt hat, und der sich heute wieder alle Mühe gibt, die mühsam gegen die Prognose von Kulturpessimisten errungene Gesellschaftsfähigkeit durch das Zurücksinken auf Kintopniveau zu verspielen. Schon heute gibt es - gewiß zu Unrecht - viele Stimmen, die behaupten, man könne nicht mehr ins Kino gehen. Die FSK ist an diesem Niveauverlust nicht unschuldig.

Gewiß darf nicht verkannt werden, daß es auch auf anderen Gebieten, etwa in der Literatur, im Theater, vor allem im Zeitschriftenwesen, einen ähnlichen - ebenfalls kommerzbestimmten - Niveauverfall gibt, daß sogar die These aufgestellt wird, allein das Triviale sei das echte Leben, während alles andere als "Schmus" abgetan wird. Doch sollte sich die FSK bei der Erfüllung ihrer Aufgabe nicht so leicht verunsichern lassen. Immerhin vermag das negative Beispiel der Selbstkontrolle der Illustrierten aufzuweisen, wohin es führt, wenn eine solche Institution zur Selbstbeschränkung dem hemmungslosen Kommerzdenken Tor und Tür öffnet. Diese Selbstkontrolle hat sich zu Tode gerichtet. Die Filmselbstkontrolle ist bei ihrer Gratwanderung zwischen Nachgeben und Noch-Festhalten an den Grundsätzen auf dem besten Wege dahin.

Ein der Prüderie gewiß unverdächtiger Zeuge, Klaus Hebecker, schrieb im „FilmTelegramm“ (Nr. 20/1970 vom 12. 5. 1970): „Ich habe während der ersten vier Monate dieses Jahres mehr Brutalität, Blut, Perversität und nackte Männer von vorn und hinten vorgesetzt bekommen als in den vorhergehenden 25 Jahren als Filmjournalist. Zur Bewältigung der in diesem Hollywood-Frühling der Presse vorgeführten Filme gehört ein Magen aus Eisenbeton, eine unerschütterliche Gefühls- 
roheit und die Fähigkeit, alles Gelernte, alle đsthetik und alle Kultur, mit der man aufgewachsen ist, abschütteln zu können, sowie gleichzeitig unfaßliche Talentlosigkeit, Selbstverherrlichung und Verhöhnung aller menschlichen Werte herunterzuschlucken. Dieser Aufgabe fühle ich mich nicht gewachsen". Hebecker könnte diese Aussage 1971 nur verstärken und auf keinen Fall abschwächen.

Ein indirektes Kriterium für die Situation des Films ist die Statistik. Sie besagt, daß 1958, dem ersten vollen Jahr nach Inkrafttreten der Novelle zum Jugendschutzgesetz, nur 14,8 Prozent der freigegebenen Filme den über 18-jährigen vorbehalten waren. Die Kurve steigt stetig an und war 1970 bei 48,8 Prozent angelangt. Dagegen sank die Zahl der familiengerechten Filme ab 6 Jahren - bei allem Vorbehalt für diese Klassifizierung - von 15,2 Prozent im Jahre 1958 auf 8,4 Prozent im Jahre 1970. Das ist die genau gegenteilige Entwicklung. Sie läßt sich nicht mit dem Hinweis auf die "strengste Jugendzensur der Welt" begründen, wie das manchmal versucht wird. Auch das ist vielmehr Zeichen für die Krise des Films, für den Versuch, die Konkurrenz des Bildschirms mit fernsehunspezifischen Brutalitäten und Sexualitäten zu schlagen. Die FSK gibt diesem Trend nach.

Aber die FSK ist emsig bemüht, ihre Wirksamkeit zu belegen. Judengschutzmitarbeiter, Juristen, Abgeordnete, der Bundesfamilienminister, ja der Bundespräsident bekamen die Schnittrollen der FSK zu sehen, jene Schnitte also, die von den Ausschüssen als Voraussetzung zur Freigabe gemacht worden und von den antragstellenden Firmen als Beweis für die Ausführung der Auflage bei der FSK deponiert worden sind.

Nicht wenigen Zuschauern kam bei diesen Vorführungen das Schaudern, und manche haben den Vorführraum verlassen. Niemand wird diese Operationen bedauern, zumal künstlerisch bemerkenswerte Filme - und seien sie noch so fragwürdig wie "Flesh" und "Trash" aus der Warhol-Faktory - ungeschoren davon zu kommen pflegen. Aber bei den Pflichtübungen der FSK zur Aufbesserung ihres Images in der Offentlichkeit sollte auch das andere Experiment gemacht werden, nämlich $\mathrm{zu}$ zeigen, was bei mühsam geretteten Filmen alles nicht geschnitten worden ist, zumal wenn der Autor einen Namen - wie etwa Kolle - hat.

Die Tatsache, daß die Grenzen des Freigegebenen in den letzten beiden Jahren immer weiter hinausgeschoben worden sind, zwang die von den Kirchen entsandten Prüfer in der FSK zu verstärkten Reflexionen über den Sinn ihrer Mitarbeit. Von ihnen, von ihren Kirchenleitungen und aus der kirchlichen wie nichtkirchlichen Offentlichkeit wird immer dringender die Frage nach dem Sinn einer weiteren Mitarbeit gestellt. Wenn die Grundsätze immer großzügiger gehandhabt werden, dann ist diese Frage vollauf berechtigt.

Nun ist die FSK - das ist mit aller Hochachtung festzustellen - eine beachtliche demokratische Selbstverwaltungseinrichtung. Wenn sich ein ganzer Wirtschaftszweig einer solchen Selbstkontrolle unterzieht und sich dazu noch im großen und ganzen äußerst diszipliniert verhält, kann das nur Respekt erwirken. So sollte bei allem Unmut über die tatsächliche Spruchpraxis die Frage gestellt werden, ob diese Einrichtung nicht erhalten bleiben kann, zumal sie sich jüngst neue (zeitgerechtere?) Grundsätze gegeben hat.

Welche Voraussetzungen wären dabei zu erfüllen?

1. Die neuen, am 1. Januar 1971 in Kraft getretenen Grundsätze, die am Grundgesetz und an den darin garantierten schutzwürdigen Grundrechten orientiert sind, 
müssen ernst genommen werden. Allerdings hat die Erfahrung der ersten Monat gezeigt, daß auch mit neuen Prüfkriterien die Prüfpraxis nicht zu ändern ist. Der allgemeine Trend einerseits und die harten Wirtschaftsinteressen andererseits stehen einer Besserung der Situation und einer neuen Glaubwürdigkeit der FSK im Wege.

2. Ebenfalls bei der Feiertagsfreigabe gibt es Wünsche. Da der Feiertagsschutz durch die entsprechenden Gesetze Sache des Staates ist, hat das Land Nordrhein-Westfalen cine Liste von - freilich nicht in jedem Fall beweiskräftigen - Titeln aufgestellh, bei denen es um den Feiertagsschutz nicht gut bestellt ist. Die Massierung der entsprechenden Feiertage im November macht die Programmgestaltung für die Filmtheaterbesitzer tatsächlich schwierig. Es sind zu wenig Filme da, die dem Charakter der Tage entsprechen. Doch ist das kein Grund dafür, daß mancher Film durchgeboxt wird, und sei es bei Stimmengleichheit mit der Stimme des Vorsitzenden.

3. Eine wichtige Verbesserung im Verfahren ist die sogenannte vorsorgliche Berufung der überstimmten Minderheit. Sie wird nur wirksam, wenn auch der Antragsteller in die Berufung geht. Die Berufungsinstanz wird dadurch meist freigestellt und muß nicht nur über die strittigen Szenen verhandeln. Sie kann also auch zusätzliche Schnitte verfügen.

Aber damit ist immer noch keine vollständige rechtliche Gleichstellung der Ausschußmitglieder gegenüber dem Antragsteller gewährleistet. Während der Antragsteller unbefristet Berufung einlegen kann, bleiben der überstimmten Minderheit ganze 24 Stunden. Die Einspruchsfrist müßte also angemessen verlängert werden. Ein kürzlid durchgeführtes Berufungsverfahren mit einem 1958 geprüften Film kann das Kuriose dieser Situation erhellen.

4. Die FSK klagt über chronischen Geldmangel. Wen wollte das wundern, wenn er weiß, daß mit einer Prüfgebühr sämtliche Instanzen, also Arbeitsausschuß, Hauptausschuß und Rechtsausschuß angerufen werden können. Außer den normalen Reiseund Aufenthaltskosten für die Ausschußmitglieder müssen unter Umständen auch Honorare und Spesen für Gutachter gezahlt werden. Ein erheblicher Aufwand, selbst wenn die Sitzungsgelder für die Prüfer vergleichsweise enorm niedrig, nämlich bei 30,- DM pro Tag, liegen.

Die Offentliche Hand - also Bund, Länder, Kirchen und Bundesjugendring - rührte die Geldnot der FSK. Was vor Jahren bereits vorübergehend praktiziert worden ist, wurde jetzt als Regel akzeptiert: Die Ausschüsse wurden verkleinert. So wurde die Mindestbesetzung im Arbeitsausschuß - sieben Prüfer - zugleich zur Normalbesetzung. Das bedeutet aber, daß der einzige hauptamtliche Prüfer der Offentlichen Hand, der Vertreter des Bundes, jede dritte Woche ausfällt. Damit ist bei zwei hauptamtlichen Prüfern der FSK - kein Prüfer der Offentlichen Hand mehr in der Lage, die Kontinuität der Prüfpraxis verfolgen zu können. Diese innere Schwächung der Position der Offentlichen Hand in der FSK haben die für diese Neuregelung Verantwortlichen unverständlicherweise nicht gesehen. Sind sie so praxisfremd?

Bei der Filmbewertungsstelle Wiesbaden (FBW) ist es üblich, daß für das Verfahren im Hauptausschuß einmal Prüfgebühren entrichtet werden müssen. Dadurch ist jeder Antragsteller gezwungen, scharf zu überlegen, ob sein Einspruch auf Revision eines Gutachtens auch begründet ist. In der FSK-Praxis dagegen ist es so, daß der Antragsteller, weil er kein Risiko zu tragen hat, auf jeden Fall nur gewinnen kann. Daher die große Zahl der Hauptausschußverfahren. Mit einer revidierten Gebührenordnung 
könnten erhebliche Mittel eingespart werden, die nur zu einem geringen Teil für die bisherige Normalbesetzung aufgewandt werden müßten.

5. Erwägenswert wäre auch die Veröffentlichung eines Minderheiten-Votums. Einer derartigen Veröffentlichung der Abstimmungsergebnisse gegenüber ist allerdings Skepsis angebracht. Da sich der aufmerksame Beobachter häufig des Eindrucks nicht erwehren kann, daß bei den Entscheidungen handfeste Wirtschaftsinteressen eine Rolle spielen, muß befürchtet werden, daß sich diese Einflüsse verstärken, wenn öffentlich erkennbar wird, wer wie gestimmt hat. Immerhin gibt es gute Gründe für die Veröffentlichung der Abstimmungsergebnisse.

6. Ein alter Wunsch an die Spruchpraxis der FSK ist die Brücksichtigung von trendbestimmenden Details (Sex, Brutalität, Aggressionen usw.). Solche Effekte werden von den Filmproduktionen oft bewußt eingebracht, um die Ausschüsse zu einer weitergehenden Spruchpraxis zu bringen. Als Beispiel können die Kolle-Filme dienen, in denen eine Eskalation der "gebrochenen Tabus" feststellbar ist. Während sich Kolle in seinem zweiten Spielfilm noch dagegen wehrt, einen Coitus darzustellen, weil er das nicht für nötig halte, da jedermann wisse, wie er vollzogen würde, schwelgt er in seinem übernächsten Film in coitalen Szenen. Die Reklame für einen späteren Film bestätigt unmißverständlich: „Mit jedem Film geht Kolle einen Schritt weiter ... Kolle geht wirklich mit jedem Film einen Schritt weiter. ${ }^{\alpha}$ In diesem Falle hat die Werbung sogar recht. Einer derartigen, sich stetig fortsetzenden Ausweitung der Spruchpraxis kann nur durch ein stärkeres Festhalten am ursprünglichen Sinn der Grundsätze entgegengewirkt werden.

Von Seiten der Filmwirtschaft wird argumentiert, daß etwa beim Film „Die Technik der körperlichen Liebe ${ }^{\alpha}$ ein solcher hinhaltender Widerstand geleistet worden sei. Drei Ausschüsse der FSK (Arbeits-, Haupt- und Rechtsausschuß) hatten die Freigabe des Films abgelehnt. Ein Gerichtsbeschluß in Berlin habe jedoch die Grundlagen verändert. So sei dieser Film bei einer erneuten Prüfung mit nur wenigen Schnitten doch freigegeben worden.

Es darf sicher nicht verkannt werden, daß die FSK durch das Berliner Urteil in eine schwierige Situation gekommen ist. Dennoch bleibt die Frage offen, ob die Argumentation: "Wenn die nichts dagegen haben, haben auch wir nichts dagegen ", nicht die Krise der FSK verschärft. Der Film hätte aufgrund der FSK-Grundsätze auch nach dem Berliner Urteil nicht freigegeben werden dürfen, auch wenn es zahlreiche Bundesbürger gibt, die diesen Film ansehen. Die FSK hätte in diesem Fall ein Exempel statuieren können. Der Trouble wäre groß gewesen, aber die Zukunft wahrscheinlich klarer.

7. Nach wie vor stellt sich die Frage nach der Qualität der Prüfer in der FSK. Viele Prüfer sehen ihre Aufgabe darin, festzustellen, ob sie selbst von dem zu prüfenden Film berührt werden oder nicht. Sie übersehen aber, daß sie nicht für sich selbst zu urteilen haben, sondern für die bundesdeutsche Offentlichkeit. Ferner fehlt ihnen vielfach die Nähe zur Jugend. Manche Vermutungen über das Verhalten der Jugend ähneln eher den Vorstellungen von Klein-Moritz als dem Urteil filmkundiger Persönlichkeiten.

8. $\mathrm{Zu}$ beklagen ist auch die Tatsache, daß Filme vielfach sehr punktuell beurteilt werden. Obwohl die FSK völlig anders als die amerikanische Selbstkontrolle strukturiert ist - sie ist eine Wirkungskontrolle und beurteilt nicht Fakten, wie es der amerikanische Production-Code vorschreibt -, wird weithin lediglich über Details 
und nicht etwa über Tendenzen geurteilt. Selbst Filme, die - wie man sagt - ,in der Wolle gefärbt sind“, werden nicht vom Grundsätzlichen her beurteilt, sondern im Detail mit der Schere behandelt. Die Ergebnisse sind dann entsprechend unbefriedigend.

9. $\mathrm{Zu}$ beklagen ist auch eine Inkonsequenz der Spruchpraxis. Offensichtliche Fehl. entscheidungen werden zur Norm erhoben. Der Hinweis: „Das ist auch in dem Film X freigegeben “, setzt dann den Maßstab für die Beurteilung des Films Y. Auch damit ist eine ständige Ausweitung der Spruchpraxis gegeben.

Sollte der Trend zur Ausweitung der Spruchpraxis nicht zu stoppen sein, wäre die Frage zu stellen, ob die FSK nicht bestimmte Filme aus dem normalen Prüfvorgang herausnehmen und mit einem Kennzeichen versehen sollte. Diese Filme könnten dann nach einer entsprechenden Vereinbarung mit der Filmtheaterwirtschaft in ganz bestimmten Häusern eingesetzt werden. Das entspräche etwa der Praxis der amerikanischen Selbstkontrolle, die auch gewisse Filme nicht erfaßt. Diese Filme werden in bestimmten Kinos mit dem Hinweis suncensored " (unzensiert) gezeigt. Allerdings dürfte dieses Zeichen nicht etwa den Werbeeffekt haben wie das " $S^{\star}$ im gleichschenklichen Dreieck, das der Avis-Filmverleih für bestimmte Filme entwickelt hat. Dieses Zeichen - so heißt es in der Werbung - „klassifiziert diesen Film deutlich als ,scharfen' Sexfilm. Wir möchten alle Besucher darauf aufmerksam machen, damit sie sich hinterher nicht sittlich entrüsten." Dieses Werbungsmittel ist selbstverständlich von der FSK nicht freigegeben.

Alle diese Verbesserungsmöglichkeiten aber bieten nur eine vage Hoffnung, daß die Rückgraterweichung der FSK geheilt werden kann. Dementsprechend sind die Kirchenvertreter in der FSK verpflichtet, ihren Auszug vorzubereiten. Sollte die FSK dadurch fallen, so müßte die Prüfung für die Jugendfreigabe auf jeden Fall erhalten bleiben. Zur Mitarbeit auf diesem noch relativ gut funktionierenden Sektor sind die Kirchen bereit. Eine Weiterarbeit in der FSK aber ist nur möglich, wenn der Eskalation von Brutalität, Gewalt und Sexualität Einhalt geboten wird.

Allerdings wird der Druck auf die FSK zu größerer Liberalität auch immer stärker. Der Druck kommt nicht allein von außen; er geht bis in die Ausschüsse hinein. Dort sind Plädoyers für die Freigabe eines Films oder die Aufhebung von Schnitten zu hören, die kaum anders als ein mehr oder minder gelinder psychologischer Druck auf die Ausschüsse zur abermaligen Ausweitung der Spruchpraxis aufgefaßt werden können. Auch unter diesem Aspekt fragen sich die Kirchenvertreter, ob sie weiter mitarbeiten können.

Die FSK ist eine freiwillige Einrichtung der Filmwirtschaft. Sie hat sich in ihrer 20jährigen Geschichte durchaus Verdienste erworben. Die Filmwirtschaft hat die Offentliche Hand zur Mitarbeit gebeten. Die von den Kirchen entsandten Prüfer haben sich für die Prüfarbeit gern zur Verfügung gestellt. Wenn aber die Effektivität ihrer Arbeit immer stärker auf die Null-Marke zu sinkt, muß der Schlußstrich gezogen werden. Die Kirchen sind FSK-müde geworden.

Damit bleibt die Idee einer demokratischen Selbstverwaltungseinrichtung in unserer Gesellschaft unangetastet. Aber dieses Instrument hat im Laufe der Entwicklung seine Wirksamkeit stark eingebüßt. Ob es seine ursprüngliche Wirksamkeit einmal wiedererlangt? Trotz Neufassung der Grundsätze und trotz aller gemeinsamen Bemühungen gibt es nur wenig Hoffnung. Die Praxis widerlegt die Theorie. Die Diskrepanz zwischen ihrem Auftrag und dem Druck der Filmwirtschaft reibt die FSK auf. Es ist schade um sie. 


\section{S U M M A R Y}

Wilhelm Bettecken describes the role of the Churches in the Voluntary Censorship of Filmbusiness (Freiwillige Selbstkontrolle der Filmwirtschaft - FSK) in Germany. He briefly outlines the history of this institution and regards the present possibilities and the decisionmaking influence of the Churches as very restricted.

The film industry takes unfair advantage of the participation of the Churches in this body in order to apologize the release of questionable movies. The author takes the view that the Churches very soon will renounce their collaboration. In conclusion he names the definite conditions for a continuation of useful co-operation.

\section{RESUMEN}

Wilhelm Bettedsen describe la posición de las Iglesias en la voluntaria autovigilancia del comercio cinematográfico en Alemania (Freiwillige Selbstkontrolle der Filmwirtschaft in Deutschland - FSK). Da un resumen de la historia de esta institución. Al presente ve las posibilidades de las Iglesias muy limitadas. Tienen apenas influencia en las decisiones y están usadas por el comercio cinematográfico como coartada para el desembargo de películas dudosas. En la opinión del autor derogan su colaboración pronto. Terminando indica en 9 artículos las condiciones de una continuada cooperación conveniente.

\section{Zur Kommunikationssoziologie des Katholizismus}

\section{von Joachim Westerbarkey}

\section{Zur Fragestellung}

Die Publizistikwissenschaft als Wissenschaft sozialer Kommunikation kann sich bei der Betrachtung religionspublizistischer Phänomene nicht mit dem "Wesen" des Religiösen beschäftigen, sondern sie interessiert sich für die aktuellen Bedingungen und Folgen des religiös-kommunikativen, speziell des kirchenpublizistischen Handelns. Sie prüft die sozialen Erwartungs- und Handlungsstrukturen z. B. kirchlicher Mitglieder in ihrer kommunikativen bzw. publizistischen Relevanz. ${ }^{1}$

Die vorliegende Studie skizziert den katholischen Klerus als kollektiven Kommunikator. Eine Kommunikatoranalyse stellt die Frage nach „den institutionellen Trägern, den Herrschaftsbefugnissen und Herrschaftsverteilungen, den Organisationsformen, den Produzenten und den Interessengruppen ..., die möglicherweise hinter den Herstellern und den Verteilern stehen ".."

Es ist eine Methode zur Ermittlung von Produktion und Produktionsnormen der publizistischen Aussage, eine publizistische Betriebssoziologie des Kommunikators und seiner Organisation. Dabei können die jeweiligen Systemstrukturen nicht unkritisch als gegebener analytischer Bezugsrahmen behandelt werden, sondern müssen selbst Gegenstand der Untersuchung sein, d.h. erst eine Analyse der klerikalen Kommunikationsstrukturen kann Aufschluß darüber geben, welche Informationsleistung sie für die klerikale Organisation, für die katholische Kirche und für die Gesamtgesellschaft $\mathrm{zu}$ bringen vermögen. ${ }^{3}$

Dr. Joachim Westerbarkey ist Wissenschaftlicher Assistent am Institut für Publizistik der Westfälischen Wilhelms-Universität Münster. 
Der funktional-strukturelle Ansatz in der Systemtheorie bietet sich als Möglichkeit an, den Klerus als Kommunikationssystem bzw. Kommunikator-Organisation einer vor allem vergleichenden Darstellung zugänglich zu machen. Die dialektische und politische Implikation allen wissenschaftlichen Handelns beinhaltet allerdings, daß jeder ideologisch noch so "unverdächtig“ erscheinende Vergleich eine Wertung durch das spezifische Erkenntnisinteresse des jeweiligen Wissenschaftlers einschließt, expressis verbis oder nicht. Das betrifft auch den Umgang mit dem Begriff der "funktionalen Aquivalenz", wenn er nicht als reine Formalkategorie verstanden werden soll; die Gegenüberstellung struktureller Alternativen unter bestimmten "funktionalen " Gesichtspunkten erscheint deshalb nur im Zusammenhang mit der Erklärung von Erkenntniszielen sinnvoll. Die Ziele der vorliegenden Skizze entsprechen den gesamtkirchlich sozialen Maximen der strukturellen Dezentralisierung, der personalen Autonomisierung zu größerer „Eigenverantwortung “ und der „funktionalen" Mitbestimmung. Die Erfüllung dieser Maximen setzt eine größere kommunikative Transparenz der klerikalen Organisation und die gesamtkirchliche Vermittlung des zur Teilnahme an der kirchlichen Entscheidungsbildung erforderlichen Wissens voraus.

\section{Kirchenbürokratie und klerikale Herrschaft}

Die römisch-katholische Kirche gilt seit eh und je als Prototyp einer hoch institutionalisierten und zentralisierten Organisation. Sie zeichnet sich aus durch einen hierarchisch strukturierten und von laikaler Kontrolle weitgehend unabhängigen Klerus, durch ein ausgefeiltes System soziokultureller Riten und Gesetze, das mit der Betonung von Herrschaft und Gehorsam verknüpft ist, und durch eine kommunikativ verschlossene und konservative bürokratische Spitze, nämlich die römische Kurie, die ihrerseits zum Symbol einer traditionsgebundenen Organisation geworden ist. Alle widhtigen Entscheidungen in der Kirche werden in hierarchischer Sukzession von diesem Herrschaftszentrum aus vermittelt. ${ }^{5}$ Dabei ist von besonderem Interesse, daß die Bezeichnung "kleros" ursprünglich die "Leute Gottes" meinte, aber in der christlichen Geschichte schon früh auf diejenigen Mitglieder der Religionsgemeinschaft angewandt wurde, die sich auf die Pflege von Lehre und Kult spezialisierten. Die zusätzlich anfallenden Verwaltungsaufgaben gingen mit dem Anspruch der Einheit von Herrschaft bald ebenfalls in die Hände der kirchlichen Elite über.

Dieser Umstand begründete die bürokratische Tendenz der klerikalen Organisation, deren zielorientiertes Handeln seitdem stets mit Verwaltungsaufgaben verbunden ist. So entstand eine Beamtenschicht in einer „Instanzenhierarchie ${ }^{\text {, }}$, die die kirchliche Herrschaft ausübt. ${ }^{8}$

Das Kriterium der geistlichen Organisation ist die Kombination von Weihe- und Amterhierarchie. Sie umfaßt die Herrschaftsdifferenzierung des Klerus und ist verbunden mit einem formalisierten System normativer Kontrolle, die ebenfalls auf einer Kombination personaler und positionaler Rollenmerkmale beruht (Etzioni) und sich primär auf expressives Kommunikationsverhalten richtet. Die Kontrollmittel der katholischen Kirche beschränken sich im wesentlichen auf normative "Symbole"; sie werden gewöhnlich durch Uberzeugungsstrategien und Sanktionen eingesetzt, wobei die soziale Wertschätzung dieser Symbole für den Erfolg der Kirche entscheidend ist. ${ }^{7}$

Klerikaler Einfluß beruht grundsätzlich auf dem Glauben der beeinflußten Partei (also der Laien) an die Legitimität der beeinflussenden und nicht auf deren Legi- 
timation durch Nachweis von Expertenschaft, d. h. von besonderem Fachwissen. Klerikaler Einfluß ist also Herrschaft und nicht „funktionale Autorität ${ }^{\text {" }}$ auf der Basis von Wissen. Die katholische Kirche bzw. ihr Klerus weist sich selbst nachdrücklich als "Herrschaftsverband " aus. ${ }^{8}$ Sie ordnet ihr eigenes "Gemeinwohl " nach Strukturmerkmalen von "Autorität" und Hierarchie; ${ }^{*}$ denn an der Spitze von Klerus und Kirche steht die päpstliche Lehrautorität. Von dort aus hat die Hierarchie „kraft ihres Amtes" die Verteilung aller Kompetenzen zu vollziehen. Aus der christlichen Verpflichtung, ihrer Ethik in der ganzen Welt Gültigkeit zu verschaffen ("Heilsaufgabe"), leitet die Kirche darüberhinaus den Anspruch einer normativen Herrschaft in der menschlichen Gesellschaft ab; sie versucht "Normen durchzusetzen, die sowohl die Tätigkeiten innerhalb wie außerhalb ihrer Organisation bestimmen ${ }^{\text {. }} .10$

Der Klerus betrachtet sich elitär als „Träger ${ }^{\alpha}$ der kirchlichen Moral, die im katholischen Dogmatismus die Form eindeutiger und Allgemeingültigkeit beanspruchender Handlungsvorschriften annimmt. Er rekrutiert ausschließlich Mitglieder, die eine auch von der Umwelt anerkannte Ausbildung vorweisen können, beschränkt sich auf wenige zusätzliche Selektionsmechanismen (Geschlecht und Aufnahmeweihen; letztere werden aber de facto nicht zur Selektion benutzt) und betont statt dessen eine intensive Indoktrination während der Ausbildung. ${ }^{11}$

Priesterschaft und Hierarchie halten offiziell die „Schlüssel ${ }^{\text {“ }}$ zur kirchlichen Tradition, zur sakramentalen Vermittlung und zur kirchlichen Rechtsprechung. Ein Zweifel an ihren Lehraussagen erscheint innerhalb der Kirche grundsätzlich ausgeschlossen: im Laienbegriff spiegelt sich die angebliche Inkompetenz der „übrigen“ (d. h. der Masse der) Gläubigen zur Kritik an Entscheidungen der Hierarchie. Entsprechend geht das Kirchenrecht allein von der klerikalen Amterhierarchie und ihrer Territorial- und Personalstruktur aus, während laikale Sozialstrukturen wie die Gemeinde juristisch nicht eigens berücksichtigt werden und (bisher) entsprechend wenig Einfluß auf die formale innerkirchliche Entscheidungsbildung haben - es sei denn, es wird ihnen ausdrücklich oder stillschweigend vom Klerus ein Mitspracherecht eingeräumt. ${ }^{12}$ Die Organisationsstruktur der katholischen Kirche trägt also immer noch „zum Teil

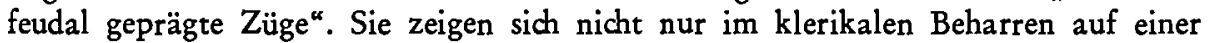
territorialen Seelsorge-Organisation, sondern generell in den als „klerikal-monarchisch-absolutistisch" charakterisierten Herrschaftsformen in Gemeinde, Diözese und Gesamtkirche, die Greinacher beinahe grotesk anmuten, wenn er bedenkt, daß die katholische Kirche z. B. in der Gesamtgesellschaft der Bundesrepublik nur eine Minderheit erfaßt, sich also vielfach in einer Diasporasituation befindet.13

Als normative Organisation hat die Kirche ein politisch-publizistisches Ziel, das sich in dem öffentlichen Geltungsanspruch ihrer spezifischen soziokulturellen („sittlichen ") Wertordnung dokumentiert. Um die Komplexität ihrer publizistisch bzw. politisch konkurrierenden Umwelt nach dem Kriterium der Gewinnung von eigenem Einfluß zu reduzieren, arrangiert sich die Amtskirche deshalb möglichst weitgehend mit den herrschenden Gruppen von Staat und Gesellschaft. Diese Anpassungsstrategien der Kirche werden durch die weitreichende politische Indifferenz ihres „Gemeinwohlprinzips ${ }^{\alpha}$ erleichtert. ${ }^{14}$

\section{Kommunikation und Entscheidungsbildung im zentralisierten klerikalen Netz}

Der Klerus läßt sich als komplexes, offenes Informations-Entscheidungs-System weitgehend von seinen kommunikativen Erwartungsmustern und Handlungen her 
begreifen. ${ }^{15}$ Die hierarchische Organisationsstruktur (Herrschaftsstruktur) des katholischen Klerus ist durch den Primat formal abwärtsgerichteter Kommunikation gekennzeichnet, also durch institutionalisierte vertikale Kanäle der Instruktion, Koordination und Kontrolle. ${ }^{16} \mathrm{Je}$ straffer eine Hierarchie organisiert ist, desto schwächer scheint der Fluß aufwärtsgerichteter Informationen zu fließen. In der hierarchischen Struktur des Klerus dienen Untergebene primär zur Rezeption und Ausführung; deshalb beschränkt sich ihre Aufwärtskommunikation meist auf die unmittelbaren Vorgesetzten. ${ }^{17}$

Im hierarchischen klerikalen Kommunikationssystem steigt die kommunikative Kapazität des Mitgliedes im Regelfall mit seiner hierarchischen Position. Die hierarchische Struktur ermöglicht es den Inhabern der Spitzenpositionen, das gesamte formale Kommunikationssystem des Klerus zu kontrollieren. Entsprechend ist das

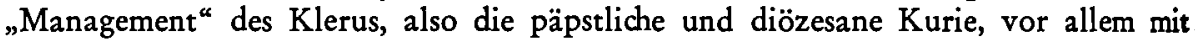
der Zielsetzung und der Kontrolle über die Zieladäquanz der Handlungen im System beschäftigt: es strukturiert, differenziert und koordiniert qua Entscheidung die Aktivitäten der Mitglieder immer im Hinblidk auf eine komplexe Umwelt, mit

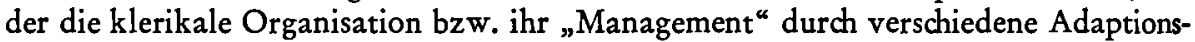
strategien verfährt. ${ }^{18}$

Insofern benötigt das klerikale "Management" die größte Kommunikationskapazität der Organisation, um direkt oder mittelbar jedes geistliche Mitglied fortlaufend mit allen Informationen versorgen zu können, die es zum systemrationalen Handeln braucht.

Durch das Prinzip des mehrstufigen Instanzenbaus (Gesamtkirche - Bischofskirche - Lokalkirche) schützt sich der Klerus auf allen Ebenen vor kommunikativer Uberlastung. Es liegt in der Uberschneidung kleíner, zentral organisierter Netze, deren Zentralstellen jeweils Untergebene in einem übergeordneten Netz sind und Wissen selektiv vermitteln. ${ }^{19}$ Geistliche als Vorgesetzte gehören (vom Papst einmal abgesehen) also jeweils mindestens zwei engeren klerikalen Kommunikationsnetzen an, die verschiedene Anforderungen an sie stellen; sie handeln daher ständig in einem möglichen oder aktuellen Konflikt. ${ }^{20}$

Alle Entscheidungen im Klerus sind abhängig von seinen Informationen, seinem Wissen; Entscheidungsbildung ist die kommunikative Schlüsselfunktion des Klerus zur Lösung aller seiner Probleme, wobei sich das klerikale System bezüglich seiner Umweltprobleme kontinuierlich selbst strukturiert. Wie die meisten (Kommunikator-) Organisationen verfügt der katholische Klerus über eine Hierarchie von Entscheidungspositionen mit formalisierten Anweisungskanälen. ${ }^{21}$ Generell trifft durch die notwendig selektive Behandlung von Information jedes Mitglied im klerikalen Kommunikationsnetz Entscheidungen, doch steigt die Relevanz der Entscheidungsbildung in den Kommunikationszentren der oberen Ränge. ${ }^{22}$

Eines der Hauptziele abwärtsgerichteter Kommunikation im Klerus ist die Überredung zu bestimmten Entscheidungen und Handlungen. Ihr Ergebnis ist trotz aller Wahrscheinlichkeit stets an den geistlichen Rezipienten gebunden, an sein Verstehen, seine Bereitschaft und seine Wertschätzung. Um die Annahme einer Norm zu erzielen, muß ein "Sender" vor allem die Legitimation besitzen, normatives Verhalten vorschreiben zu dürfen. Gewöhnlich wird der „Sender" durch Bezug auf die Normen der klerikalen Organisation und/oder durch formale Delegation durch einen Ranghöheren legimitiert. Das Schwergewicht der klerikalen Entscheidungsbildung liegt dementsprechend auf den kirchengesetzlich vorgeschriebenen Strukturen, also auf 
den formalen Mustern der Organisation, die als Rahmen möglicher Entscheidung dienen und die durch rationale situative Ubersicht und durch das gesicherte Wissen Einzelner ergänzt werden können. ${ }^{23}$

\section{Dezentralisierung durch „funktionale Autorität ${ }^{\alpha}$ : Aspekte ciner Zielvorstellung}

Niemand hat in einer Organisation genug Information, um isoliert entscheiden zu können, d. h. jeder muß sich an einem extensiven Kommunikationsprozeß beteiligen. In größeren Systemen wie dem Klerus kann deshalb und aufgrund der notwendig arbeitsteiligen Behandlung komplexer Probleme das hierarchische Zentralnetz nicht die einzige Regel faktischen Entscheidens sein.

Der gesellschaftliche Trend einer zunehmend fachlichen Spezialisierung deutet vielmehr darauf hin, daß formale Entscheidungen künftig immer mehr in horizontalen Kontakten gefunden werden müssen, während vertikale Kommunikation vor allem die Aufgabe behält, Probleme auf die richtigen Entscheidungsebenen $z u$ verteilen bzw. Entscheidungen im „Dienstweg“ zu koordinieren. ${ }^{24}$ Moderne Spezialexpertise verlangt auch vom Klerus eine dezentrale Entscheidungsbildung über sachbezogene Probleme. Dabei wird "funktionale Autorität" zum Entscheidungsprinzip fachlicher "Teams", während das hierarchische klerikale "Management" sich weitgehend auf die Definition generalisierter Entscheidungsprämissen (z. B. Mitgliedschaftsbedingungen) oder gar nur auf Koordination und generelle Erfolgskontrolle beschränken muß.

Hartmann konzipiert „funktionale Autorität“ als das genaue Gegenteil von positionaler Herrschaft bzw. von Amtsgewalt: ${ }^{25}$ der Sachverständige kann sich nie unmittelbar auf eine hierarchische Struktur berufen, sondern muß sich sters erklären und beweisen, andere also durch sein Wissen "objektiv" und direkt überzeugen. Die Beeinflußten müssen ihrerseits aktiv am Zustandekommen seiner Legitimation teilnehmen: die Form ihrer Folgeleistung besteht in der kritischen Prüfung. Sozusagen als Aquivalent dafür, „daß die funktionale Autorität das herkömmliche Muster organisatorischer Ober- und Unterordnung zersetzt", ergibt sich eine "funktionale Hierarchie“, die durch die Offenheit des Wettbewerbs um "funktionale Autorität" Stetigkeit erhält. ${ }^{2 \theta}$

Die von Hartmann geforderte Dezentralisierung von Entscheidungspositionen in Organisationen impliziert die Unterteilung des Klerus in relativ autonome oder halbautonome Entscheidungseinheiten, wobei den bisher untergeordneten Einheiten Verantwortung und Kontrollmöglichkeiten gegenüber Vorgesetzten übertragen wird. Es geht um eine im Hinblick auf das jeweilige Problem "optimale Zentralisierung" (Ziegler) der klerikalen Organisationen; in diesem Sinne ist auch das Konzept der "Mitbestimmung" zu verstehen. Durch Dezentralisierung werden vor allem Möglichkeiten abweichender Problemlösungsvorstellungen provoziert, was sich in der Diskussion um die Bewältigung aktueller Konflikte äußerst fruchtbar auswirken kann. ${ }^{27}$

Betrachtet man also "funktionale Autorität" als "funktionales Äquivalent" zu Herrschaft, dann ließe sich die Zielvorstellung des Abbaus hierarchischer Herrschaft in der katholischen Kirche quasi "ohne funktionale Verluste" zugunsten einer größeren Kommunikationskapazität der Organisation verwirklichen, d.h. klerikale Herrschaft könnte weitgehend durch kirchliche Autoritätsstrukturen auf der Basis von Wissen substituiert werden. ${ }^{28}$ Noch findet die Uberlegenheit von Experten aber 
ihre empirische Grenze in der durch traditionale und bürokratische Legitimation gesicherten Position des klerikalen Vorgesetzten, wobei die Tatsache, daß der Experte meist nur Kompetenzen für die Bearbeitung eines speziellen Aspektes komplexer Probleme beanspruchen kann, der Veränderung seines Status sehr im Wege steht.29

\section{Möglichkeiten und Grenzen klerikaler Innovation}

Die hochgradig zentralisierte Organisationsform des klerikalen Kommunikationsnetzes stellt sicher, daß genau an einem Punkt die wichtigsten Entscheidungen getroffen werden. Nach vier Hypothesen von Ziegler bedeutet das, daß der Klerus als soziale Organisation bei der Lösung bestimmter Probleme eine vergleichsweise hohe Effizienz hat. ${ }^{30}$ Hierarchisch-zentralisierte Netze sorgen im Regelfall z. B. für eine schnelle und effektive Lösung einfacher Koordinationsprobleme, weil sie die im Netz vorhandenen Informationen am besten übersehen können. Andererseits beeinträchtigt die Beschränkung zahlreicher Kommunikationsmöglichkeiten die Auswertung von Wissenspotential (Informationsbesitz) im Klerus; denn nicht alles denkbare kommunikative Handeln wird stimuliert, vor allem kein kritisches, d. h.: unter straffer hierarchischer Kontrolle gedeihen Innovationskonzepte minder gut. Tauchen deshalb komplexe Probleme auf, dann ist das zentralisierte klerikale Netz einem dezentralisierten unterlegen, das mehr Nachrichten abgibt (gemessen an der Minimalzahl der für die Organisation notwendigen Informationen) und daher eher und für die Mitglieder befriedigender komplexe Fragen löst. Allerdings muß auch ein dezentralisiertes System intermediäre Arbeitsgänge selektiver Beurteilung und Koordination ansetzen. Verfährt es nicht bewußt selektiv bzw. rational, dann hat sein hohes Innovationspotential nur eine geringe Durchführungschance. ${ }^{31}$

Bürokratische Orientierung ist immer konservativ. Dennoch gibt es nach V. A. Thompson einige Möglichkeiten und Voraussetzungen für den Klerus, Innovation zu formalisieren: er braucht genügend Innovationsquellen, einen hohen kommunikativen Input und eine starke Streuung von Ideen, die wiederum von einem hohen Grad kommunikativer Freiheiten und Techniken abhängt. Klerikale Innovation bedeutet dann eine Verunsicherung bestehender Organisationsstrukturen durch gröBere Entscheidungsfreiheiten, eine möglichst schwache Betonung der Grenzen von Rechten und Pflichten, eine Dezentralisierung des Kommunikationsnetzes und eine Status-Entgrenzung, also eine formale Abwertung von Herrschafts- und Amtspositionen. ${ }^{32}$ Die bürokratische Legitimation, die dabei zugunsten jener ,funktionalen " mehr und mehr verheimlicht wird, verliert in gleichem Maße an effektiver Geltung, wie die "funktionale" Legitimation sie substituiert. Das Mitglied der Organisation wird nunmehr offiziell berechtigt, den Rat seines Vorgesetzten nach seinem sachlichen Wert zu behandeln, d. h. deutliche Auflösungstendenzen des Vorgesetzten-Untergebenenverhältnisses werden sichtbar: "Die Arbeit an bestimmten Problemen oder doch wenigstens einzelne Aspekte dieser Arbeit werden herrschaftsfrei. ${ }^{{ }_{93}}$

In jedem Fall wird die Entscheidungsebene weiter nach unten verlagert, d. h. die Effektivität aufwärts fließender Nachrichten bestimmt zunehmend die Leistungsfähigkeit der Organisation, und spezialisierte Arbeitsgruppen übernehmen mehr und mehr die Verantwortung und die Initiative für Innovationen. ${ }^{34}$ Der provozierendste Zug der dezentralen klerikalen Ordnung liegt in jenem Bruch mit der Kontinuität im Fortbestand hierarchischer Verhältnisse, abgesehen von der "Objektivierung " der Erfolgskontrolle, die nunmehr vom Untergebenen selbst vollzogen werden kann. 
Dabei beläßt ein solcher Bruch der klerikalen Organisationsstruktur genügend Stabilität, um die Erhaltung ihrer Identität als religiöses Kernsystem zu garantieren. Die Möglichkeit einer dezentralen, aber einheitlichen Leitung erscheint insofern als "praktisches Modell“ für eine innovative klerikale Organisationsstruktur geeignet. ${ }^{35}$ Es ist allerdings illusorisch anzunehmen, daß die katholische Hierarchie angesichts ihrer aktuellen kritischen Phase unverzüglich durchgreifende strukturelle Veränderungen einleiten oder gar durchführen kann und wird.

Die grundsätzliche Schwierigkeit einer Organisation, aufgrund interner Probleme direkt einen Systemwandel $\mathrm{zu}$ vollziehen, erklärt Luhmann vor allem durch das formal-strukturelle Verhaftetsein von Organisationen, das etwa durch Indifferenz gegenüber bestimmten Umweltveränderungen der Sicherung ihrer Identität dient. Bestandserhaltung bei gleichzeitigen Konflikten und Wandel ist insofern ein Problem nicht nur der Kirche und des Klerus, sondern jeder sozialen Organisation überhaupt. ${ }^{36}$ Nach Möglichkeit werden klerikale Konflikte, die nicht in normalen Entscheidungsverfahren abgearbeitet werden können, „durch Formeln, Freundlichkeiten und Fiktionen" überdeckt. Strukturell bedingte Widersprüche können schon deshalb nicht offiziell anerkannt werden, weil Meinungsdivergenzen außerhalb einer gewissen Toleranz bzw. Indifferenz nicht formal legitimiert werden können. Diese strukturelle Einstellung der formalen klerikalen Organisation $\mathrm{zu}$ internen Konflikten veranlaßt sie zu Fehldiagnosen über sich selbst und zu besonderen publizistischen Aufwendungen „für die Darstellung der Einmütigkeit nach außen“ ${ }^{\text {.37 }}$

\section{Klerikale Anweisungspublizistik}

Alle Religionsgemeinschaften sind Kommunikationsgemeinschaften, weil Religion und Kirche nur durch Kommunikation möglich sind, durch gesellschaftlich bedingte und bedingende Vermittlung von Bedeutungen bzw. "Sinn", d.h. von Wissen. Das kirchliche Kommunikationssystem erhält seine Identität durch den spezifischen religiösen Wertakzent, den es dem soziokulturellen Wissen gibt. Als Realisator öffentlicher und aktueller Aussagen reduziert der klerikale Kommunikator das Ereignisuniversum nach den von ihm individuell und kollektiv internalisierten soziokulturellen Werten. Er produziert eine pointierte Meinungspublizistik, die in der Kirche durch Sozialisations- und "public relations"-Funktionen vorbereitet und unterstützt wird. Die Aussageproduktion der arbeitsteilig gegliederten Kommunikatororganisation „Klerus“ besitzt grundsätzlich die publizistische Qualität der Kollektivität. Die Verantwortung für das Endprodukt wird dabei geteilt, d.h. die klerikale publizistische Persönlichkeit tritt stets in gewissem Umfang hinter die kollektiv vorprogrammierte Aussage zurück. ${ }^{38}$

Grundsätzlich unterliegen die Beziehungen „zwischen Information, Struktur der Gemeinschaft und Hierarchie in der katholischen Kirche denselben soziokulturellen Bedingungen wie in anderen organisierten sozialen Systemen. $\mathrm{Da}$ die katholische Kirche organisatorisch weitgehend im absolutistischen Schema der Renaissancezeit stehengeblieben ist, fehlt ihr ein moderner Informations- und Autoritätsstil; denn auch das vom II. Vatikanischen Konzil befürwortete Mitspracherecht der Laien ist bisher ohne bemerkenswerte praktische Folgen für die kirchliche Entscheidungsbildung geblieben. ${ }^{30}$ Wie im Klerus ist der Informationsfluß in der gesamten katholischen Kirche nur in einer Richtung formal gewährleistet, nämlich von oben nach unten, wobei der Klerus als die "obere" Mitgliedergruppe nicht nur alle Entscheidungen über die kirchlichen Ziele, „direktes" und "mittelbares" Handeln trifft, 
sondern die meisten (Ziel-)Tätigkeiten auch selbst ausübt. Die Laien als "untere“ kirchliche Mitgliedergruppe sind in diesem Versorgungs- und Kontrollsystem weitgehend nur "Gegenstand" bzw. Konsumenten klerikalen Handelns. Sie kooperieren dabei mehr oder weniger freiwillig, können aber kaum mitbestimmen, ${ }^{40}$ obwohl sie den Klerus wirtschaftlich weitgehend tragen. Die Kirche ist also ein Muster für Anweisungspublizistik, in dem die Gläubigen regional gruppiert einen großen Offentlichkeitskreis bilden und zugleich die sozioökonomische Basis der gesamten Organisation erarbeiten. Die vertikale Publizistik der kirchlichen Hierarchie gleicht der Publizistik autoritärer Regierungen (ohne daß die Kirche als ganze mit diesen verglichen werden soll): es ist eine organisierte "Kommandopublizistik", in der die Aussage einseitig vom Kommunikator zum Rezipienten verläuft und in der der Rezipient in der Regel keine bedeutenden Realttionen zur Aussagequelle zurück-kommuniziert. Allerdings spielt sich auch diese autoritäre Publizistik im Rahmen eines kommunikativen Systems normierter Erwartungen ab, das nicht ohne Bedürfnisse bzw. Bereitschaften funktioniert. ${ }^{41}$ Arg betroffen von dem hierarchischen Entscheidungssystem der Kirche sind aber nicht nur die Laien, die vielfach ihr kommunikatives Dilemma gar nicht durchschauen, sondern auch die Pfarrgeistlichen, die als lokale kirchliche Führungsfiguren der informalen Kommunikation ihrer Gemeindemitglieder ausgesetzt sind und gleichzeitig ihre formale Verantwortung den Vorgesetzten gegenüber erfüllen müssen, um ihre Position zu behaupten. Die Gemeindemitglieder nehmen ideell motiviert zu ihnen Kontakt auf, während die klerikale Bürokratie vor allem instrumentale bzw. materielle Ansprüche an sie stellt. Zwischen diesen beiden Kommunikationsnetzen sollen die Seelsorger vermitteln bzw. übersetzen, was mangels formalisierter Kanäle zumindest aufwärts nicht adäquat möglich ist. Das führt dazu, daß die Seelsorger in bestimmten Streitfragen nur deshalb nicht mit ihren Gemeindemitgliedern übereinstimmen können, weil ihnen sonst die positionale Anerkennung der Hierarchie versagt bleibt. ${ }^{42}$

\section{Inkongruente kognitive Rangreiben}

Der weitgehende Verzicht auf formale kommunikative Rückkopplungskanäle in der katholischen Kirche rächt sich z. Z. besonders durch eine wachsende Inkongruenz der Bezugssysteme des Verstehens und der religiösen und sozialen Werte zwischen Klerikern und Laien und durch ihre gegenseitige Einschätzung mit vielfach emotionalen und negativen stereotypen Kommunikationsinhalten. Boyd meint generell, daß die Kirche aufgrund ihrer Herrschaftsstruktur nicht adäquat mit der modernen Gesellschaft kommuniziere. Statt Dialogen zwischen Klerus und Laien bzw. zwischen Kirche und Gesellschaft finden meist wechselseitige Monologe statt, die zahlreiche Mißverständnisse, Vorurteile (und damit Kommunikationsschranken) stabilisieren. Besonders unter Intellektuellen kursiert ein dezidiertes antiklerikales Ressentiment, außerhalb wie innerhalb der katholischen Kirche; letzteres hat seine Wurzeln vor allem in der formalen laikalen Abhängigkeit vom Klerus. Es könnte behoben werden, wenn einerseits der Klerus den Beweis z. B. soziokultureller oder sozialpsychologischer Sachverständigkeit erbrächte und damit $\mathrm{zu}$ einer "funktionalen Autorität ${ }^{\text {“ käme }}$ und wenn andererseits unter den Laien das Bewußtsein wachsen würde, zur persönlichen Mitsprache und Kritik an der Kirche berechtigt und verpflichtet zu sein.43

Sind die Rangreihen in den Erwartungen von Kommunikator und Rezipient inkongruent, dann deutet sich gewöhnlich ein Wandel im sozialen System an, begleitet von einer Veränderung der Stereotypen und einem Wandel des kommunikativen 
Handelns. In der katholischen Kirche ist eine solche Inkongruenz kommunikativer Rangreihen evident; ${ }^{44}$ gleichzeitig verdeutlichen zahlreiche kirchliche Statistiken die aktuelle Bestands- und Funktionsproblematik des Klerus. Wachsender Nachwuchsmangel, Überalterung und eine deshalb steigende Aufgabenüberlastung des Klerus einerseits und eine zunehmend problematische Umwelt durch den kontinuierlichen Rückgang kirchlicher Laienaktivitäten andererseits sind zu verzeichnen. Dahinter steht ebenfalls jenes kontinuierliche Auseinanderfallen formalkirchlicher (klerikaler) und sozialkirchlicher bzw. gesellschaftlicher Erwartungsstrukturen im Katholizismus bezüglich religiöser Werte und kirchlicher Normen bzw. Organisation.45

Zwar läßt sich z. B. die kirchliche Medien-Bewertungspraxis theologisch aus der Sittenlehre legitimieren; denn die letzte Identität von christlicher Seins- und Sollensordnung macht die christliche Ethik zu einem Politikum, d. h. alles zumindest klerikale Handeln impliziert eine entsprechende Wertung. Doch einerseits werden alle sittlich religiösen Bewertungen auf mögliche Wirkungen der Medien hin getroffen - ein Ansatz, der angesichts der Ergebnisse moderner Sozialpsychologie in dieser Form nicht mehr vertretbar ist - , und andererseits werden publizistische Bewertungssysteme heute vielfach als entmündigend und autoritär empfunden.46 Im Zeichen eines bedeutenden innerkirchlichen Wandels publizistischer Erwartungen wird also die normative Vorwahl ethischer Entscheidungen problematisch. Es entstehen rezeptive Dissonanzen vor allem bei den Laien, die neue kognitive Mechanismen bzw. Strukturen zur Bewältigung publizistischer Probleme suchen, da diese Probleme durch rein ethische Kategorien nicht hinreichend erklärt werden können. Auch das sind eindeutige Zeichen einer kirchlichen und klerikalen Krise, in der die gesamte formale kirchliche bzw. klerikale Organisation (Normen, Rollen und Institutionen bzw. Herrschaftsverhältnisse) solange verunsichert werden wird, bis neue, d.h. den Umweltproblemen adäquatere Strukturen formale Relevanz gewinnen. Wechselseitige Lernprozesse zwischen Klerikern und Laien mit dem Ziel neuen kommunikativen Handelns sind deshalb die Voraussetzungen zur Verminderung der erheblichen Spannungen, die zwischen den traditionellen kirchlichen Anforderungen hinsichtlich Glaube, Sitte und kirchlicher Disziplin und einem zunehmend kritisch reflektierenden Glauben unter den Laien bestehen. ${ }^{47}$

\section{Klerikale Hierarchie, Kollegialität und „kirchliche Demokratie"}

Alle Mitglieder der katholischen Kirche sollten (Golomb) die Möglichkeit haben, ihr eigenes organisationsrelevantes Informationspotential in die kirchliche Entscheidungsbildung miteinzubringen; dann könnten die meisten kirchlichen Probleme direkt und empirisch "von unten" eingebracht, diskutiert und gelöst werden. Solange aber das offizielle Leitbild der Kirche eine durch doktrinäre Reduktion von Problemen künstlich geschaffene Harmonie zu sein scheint, werden ihre aktuellen Bestands- und Entwicklungsprobleme verschleiert statt gelöst. ${ }^{48}$

Der theologische Begriff des Dienstes verhindert immer noch häufig die Einsicht, „daß faktisch die Kirchenleitung Macht ausübt “. Die Bevormundung der Gläubigen ist aber ein Herrschaftsstil, der modernen sozialen Zielvorstellungen widerspricht. Deshalb ist es nötig, sich ernsthaft auf die gesellschaftliche Bedingtheit der kirchlichen Autorität zu besinnen, nachdem bereits das Konzil eine Anpassung der kirchlichen Autoritätspraxis an die modernen Verhältnisse gefordert hat. Doch der Amtskirche fällt es sichtlich schwer, derartige Weisungen des Konzils zu verwirklichen. ${ }^{4 \theta}$ 
Mit der Offnung der in der Hierarchie dargestellten reinen Amtskirche zum „Volk Gottes" soll die traditionelle Herrschaftskirche vom Prinzip der "Kollegialität" durchdrungen werden, vom „Geist der Brüderlichkeit und des Dienstes“ ${ }^{\star 50}$. Golomb bezeichnet die partnerschaftliche und kollegiale Struktur, die neuerdings in der kirchlichen Planung und Diskussion eine große Rolle spielt, als die "notwendige Konkretisierung des Wesens der Kirche ${ }^{\alpha}$ in der heutigen Welt. ${ }^{51}$

Weil Kollegialität als Strukturprinzip alle Organisationsebenen der Kirche betreffen soll, muß die kirchliche "Macht" künftig stärker horizontal verteilt und die Ausübung des kirchlichen Amtes "demokratisiert" werden. Das Autoritätsproblem kann insofern auch durch die ,erhoffte Verwirklichung der Kollegialitätstheologie in der Diözese" und durch eine "theologisch saubere Reduktion kirchlichen Gehorsams auf seinen echten Bestand" weitgehend gelöst werden. Kirchliche Kollegialität als Kooperationsform will nämlich eine "nach Funktionen gegliederte Zusammenarbeit von Gleichen " auf der Grundlage der Einordnung in eine formale Organisation, die für eine komplexe Großkirche notwendig ist. ${ }^{52}$

In der Praxis geht es bei einer solchen kirchlichen "Demokratisierung “ zunächst vor allem um zweierlei; zum einen soll das Schwergewicht der Organisation von Rom auf die einzelnen Diözesen und Gemeinden verteilt werden, zum anderen soll die innere Distanz zwischen der Kirchenführung und der „Basis“, d. h. den übrigen Mitgliedern behoben werden. Auch die Theologie kann nun nicht mehr an den sozialen Dimensionen kirchlicher Autorität vorbeisehen; denn im Falle einer prinzipiellen Gleichberechtigung aller Kirchenmitglieder muß sich „funktionale Autorität" , in ständigen Verhandlungen $z$ wischen Ubergeordneten und Untergeordneten bewähren " (Hoefnagels). ${ }^{53}$ Die Schaffung eines breiten innerkirchlichen Konsenses ist deshalb nur unter Einsatz demokratischer Mittel möglich, nämlich durch eine allgemeine Teilnahme an Entscheidungen aufgrund struktureller Dezentralisierung und Autonomisierung, die wiederum auf einer sozialen Segmentierung bzw. Spezifizierung der Kirche beruht. Zwar erschwert und verlangsamt eine breite Beteiligung am Entscheidungsprozeß die Bildung von Entscheidungen und Programmen, doch sichert sie die reibungslose und rasche Exekution derselben. ${ }^{54}$ Doch obwohl sich die konziliare Betonung laikaler Eigenverantwortung und das Bekenntnis zum Ơkumenismus nicht mit dem traditionellen Herrschaftsstil der katholischen Kirche vereinbaren lassen, provoziert der Klerus weiterhin durch zahlreiche Zeichen seines ungebrochenen Zentralismus die Unzufriedenheit mit und in "seiner Kirche". Nach wie vor werden die Gläubigen zeitlebens als "Pfarrkinder" behandelt, wodurch die klerikale Aufgabenüberlastung stabilisiert wird. Statt dessen sollten die Amtsträger die Laien möglichst frühzeitig und konsequent $\mathrm{zu}$ kirchlicher Reife und Mündigkeit sozialisieren helfen, die allein ihre Mitbestimmung rechtfertigen und qualifizieren kann; denn Mitbestimmung ist ein kommunikatives Problem der Vermittlung von zur Entscheidung notwendigem Wissen.

Laikale Eigenverantwortung bestreitet übrigens nicht jede Kontrolle bzw. Koordination einer kirchlichen Hierarchie, will aber im Sinne der christlichen Soziallehre, daß die Teilbereiche der Kirche ihre Probleme zunächst selbst zu lösen versuchen. "Einheit" wird deshalb in einer "demokratisch" strukturierten Kirche zu einer ständig neu gestellten Aufgabe. ${ }^{55}$ Die ursprüngliche, hierarchische Verfassung der Kirche braucht also kein Widerspruch zur Mitverantwortung und Mitentscheidung aller Mitglieder zu sein; denn das klerikale Amt könnte sinnvoll in diesen gesamt- 
kirchlichen Entscheidungsprozeß integriert werden. Erforderlich ist in erster Linie die "Verlagerung des Schwerpunktes von amtlicher auf fachliche und menschliche Autorität ${ }^{\text {" }}{ }^{\text {sB }}$ Eine "funktionale" Spezialseelsorge z. B. soll deshalb den Primat des Territorialprinzips zugunsten des Sozietätsprinzips aufheben. Ziel dieser gruppenspezifischen Aktion ist ebenfalls die größere soziale Beteiligung der Gläubigen an der Kirche; Voraussetzung dafür ist, daß die dadurch entstehenden spezifischen Organisationssegmente bzw. -einheiten relativ und kommunikativ transparent sind. Eine solche neue Zielvorstellung für die Kommunikation in der Kirche findet theologische und soziologische Ubereinstimmung. Zwar kann die sakramentale Kirche ihre normative bzw. werthierarchische Bedingtheit nicht durch eine absolute Demokratie ersetzen, aber ihre sozialen Strukturen können konsequent „ demokratisiert ${ }^{\text {“ }}$ bzw. dezentralisiert werden.

Immer noch sind aber nicht nur der Klerus, sondern auch zahlreiche kirchliche Verbände dem Prozeß innerkirchlicher demokratischer Meinungsbildung und Mitbestimmung gegenüber befangen. Sie bevorzugen in der Regel Kommunikationssysteme ausgewählter Vertrauensleute, in denen Nachrichten manipulativ zugespielt und Meinungen an bestimmten Stellen strategisch absorbiert werden können; außerdem klassifizieren sie die Mitglieder der Kirche unbeirrt nach „Elite" und „Masse“. 57 Die mangelnde Einsicht der Kirchenleitung in gesellschaftliche Notwendigkeiten begründet darüberhinaus ihr geringes Engagement im Aufgebot von Fachexperten; hinzu kommt die allerdings berechtigte Sorge, ,diese Spezialisten könnten sich der kirchlichen Kontrolle entziehen", also zum "Machtverlust" der hierarchisch orientierten klerikalen Spitze beitragen. Ein Strukturwandel wäre dann nämlich nicht mehr aufzuhalten: „Die hierarchische Ordnung mit ihrer absoluten Weisungsbefugnis von oben nach unten wird Formen der Kooperation $z$ wischen Leitung und Spezialisten weichen." 58

Es darf resumiert werden, daß die gegenwärtigen kirchlichen und klerikalen Konflikte vorwiegend aus divergierenden Autonomie- und Kompetenz-, d.h. aus Herrschafts- bzw. Autoritätsansprüchen resultieren. Der moderne Trend sozialer Funktionsverluste des Klerus stellt seine bestehenden Norm-, Rollen- und Herrschaftsstrukturen grundsätzlich infrage und verunsichert den Bestand der gesamten Kirche. Alle publizistischen Adaptionsbemühungen etwa im Zeichen einer neuen „apostolischen Gesamtstrategie" sind deshalb ohne einen strukturellen Wandel der Klerus-Organisation sinnlos, der mit einem Wandel der gesamtkirchlichen Normen und Erwartungen dialektisch verbunden ist.

\section{Das Postulat der Transparenz in der kircblichen Krisenrealität}

Organisierte Kommunikationssysteme sind Lernsysteme in einer sich wandelnden Umwelt, die sich entweder rechtzeitig verändern oder zerfallen. Die Umwelt des Klerus ist ein Gebilde zahlreicher Interdependenzen verschiedener sozialer und personaler Strukturen. Auf der einen Seite weisen z.B. Verkündigung, Kulturgemeinschaft, Gemeindeleben oder Kirchenpresse integrativ auf einen relativ geschlossenen kirchlichen Raum hin, doch andererseits ist der Bereich der Kirche im Zeitalter publizistischer Massenmedien nicht mehr geschlossen zu halten. Die Kirche muß deshalb kommunikativ offen sein, nicht nur aus theologischen, sondern auch aus strategischen Gründen, will sie überleben. Zur zeitgemäßen sozialen Aktualität der Kirche gehört 
also ihr intensives öffentliches Handeln, dem wiederum eine umfassende Information der Offentlichkeit über die Probleme der Kirche entsprechen muß.58

Die Kirche hat schon immer über zahlreiche Kommunikationsstrategien verfügt, über "rationale Konstrukte hierarchisch geordneter Präferenzen", die soziales Handeln im Rahmen der in der Kirche allgemein anerkannten Ziele kirchlichen Handelns bezwecken. Eben diese Strategien sollten sich zunehmend an den Maximen kommunikativer Transparenz orientieren; ${ }^{80}$ denn zum Dialog zwischen Kirche und Welt gehört, wenn dieser effizient sein soll, notwendig die institutionelle Transparenz der Kirche, d.h. die formale Bereitschaft ihrer Mitglieder oder Mitgliedergruppen zur Information. Eine erhöhte oder gar grundsätzliche klerikale Transparenz zur Verbesserung kirchlicher Umweltbeziehungen könnte zumindest zwei positive Folgen haben: die konstruktive Kritik an kirchlichen Strukturen und Handlungsweisen könnte erweitert und die interne Entscheidungsbildung verändert werden. Außerdem dürfte durch die Verstärkung des innerkirchlichen Nachrichtenflusses auch die Einstellung z.B. profaner Medienorganisationen gegenüber kirchlichen Belangen verbessert werden; denn es besteht eine enge Korrelation zwischen den Beziehungen der Kirche zur Offentlichkeit und den aktuellen kirchlichen Innovationsbestrebungen. ${ }^{01}$

Die Aktivierung einer funktionsfähigeren internen Kommunikation zur Lösung komplexer Probleme und als Korrektiv aller kommunikativen Beziehungen zur Umwelt, beispielsweise aller klerikalen bzw. kirchlichen Publizistik, ist also grundlegend für eine positive gesellschaftliche Entwicklung der katholischen Kirche. Nur ein umfassender innerkirchlicher Dialog bietet die Gewähr für einen „aufrichtigen Dialog" der Kirche mit der Welt. Es ist sehr wahrscheinlich, daß die Kommunikation $z$ wischen Kirche und Gesellschaft umso besser funktioniert, je stärker und vielseitiger der interne Informationsfluß der Kirche ist. ${ }^{82}$

Nur im ständigen kommunikativen Umgang mit allen modernen soziokulturellen Problemen lernen schließlich die Mitglieder von Kirche und Klerus, relativ eigenständig und kritisch zu denken, und nur so können die bisher "unteren" Mitglieder zu echten Entscheidungen stimuliert werden. Der Kirche wird eine grundsätzliche Offenheit in ihrer Kommunikationspolitik also künftig nicht erspart bleiben, wenn sie nicht die Solidarität ihrer Mitglieder leichtfertig aufs Spiel setzen will. Noch schwankt die Medienpolitik des Klerus zwischen Extremen wie dem Willen zum kritischen Dialog und einer offenen oder verschleierten Zensur, wie der Kooperationsbereitschaft und autoritärer Repression. Auf der einen Seite wird z.B. das Recht auf „objektive“ Information als "unanfechtbares und unveräußerliches Menschenrecht" anerkannt und postuliert; doch auf der anderen Seite gehört die Kirche nach wie vor zu denjenigen gesellschaftlichen "pressure groups", die in der Publizistik sittliche Tabus geltend machen; denn bei aller technischen Wertschätzung sind dem Klerus die Massenmedien immer noch verdächtig: „Es entspricht bestimmten kirchlichen Traditionen, öffentliche Meinung und Religion als Pole der Veräußerlichung und Verinnerlichung zu verstehen und fast diastatisch gegenüberzustellen. ${ }^{\text {"03 }}$

Die Leitlinien katholischer Kulturpolitik setzt im übrigen nach wie vor allein der Papst, wenn auch gelegentlich kooperativ mit einem Konzil, und jeder Versuch einer echten Mitbestimmung der übrigen Kirchenmitglieder scheitert gewöhnlich am bestehenden Kirchenrecht, das den Laien die christlich-soziale "Mündigkeit" versagt. So bleiben die Entscheidungen des kirchlichen Lehramtes für die Laien unkontrollierbar und verpflichtend. Die daraus resultierende kirchliche Kommunikationskrise charakterisiert die aktuelle Autoritäts- und Bestandskrise der Kirchenorganisation. 


\section{$X$. Einige Thesen}

In offenen Kommunikationssystemen ist Wandel immanent. Deshalb versuchen sie, eine soziale "Ultrastabilität" zu erreichen, also ihren Bestand durch Lernprozesse und Wandel zu erhalten. Prinzipiell sind auch die katholische Kirche und ihr Klerus soziale Lernsysteme, die ständig strategisch zwischen Innovation und Routine wählen müssen; doch die Priorität von Routine charakterisiert sie als monokratische Systeme vom Typ der traditionellen Hierarchie, deren Kommunikation durch zahlreiche Barrieren eingeschränkt wird und in der die Entscheidungsbildung auf die Organisationsspitze zentralisiert ist. Solche Systeme sind typisch konservativ. Durch die Perpetuierung des status quo, also des traditionellen klerikalen Kommunikationsund Herrschaftssystems werden die aktuellen Probleme der Kirche verschleiert statt gelöst. Ihre Konfliktsituation, die auf einer wachsenden Inkongruenz kommunikativer Rangreihen vor allem zwischen Klerus und Laien beruht, erreicht dadurch im Hinblick auf den Bestand der Kirche Krisendimensionen.

Zahlreiche kritische katholische Gläubige wollen den offenen Dialog mit einem kommunikativ transparenten Klerus und die gleichberechtigte Teilnahme am kirchlichen Entscheidungsprozeß zur Steigerung ihres Einflusses und ihrer personalen Autonomie in der Kirche. Die damit verbundene theoretische Reziprozität als Zielund Idealfall gegenseitiger Kontrolle von Klerus und Laien impliziert eine wachsende Entgrenzung der hierarchischen Status- und Herrschaftspositionen in der Kirche: das klerikale Amt wird bei prinzipieller und theologisch begründbarer Gleichheit aller Gläubigen nur noch bestimmte Fachaufgaben umfassen, während die Hierarchie nur noch Koordinationszwecken zu dienen hat. Der Klerus als bisher dominanter kirchlicher Kommunikator wird dann zum Anachronismus; denn die Publizistik (z.B. als Verkündigung) der Kirche wird im Zeichen sozialer Aufgabenspezifizierung ein Sachbereich bestimmter qualifizierter Mitglieder der Gesamtorganisation. Voraussetzung zur Verwirklichung dieses Ziels sind wiederum Lernprozesse für neues kommunikatives Handeln in der katholischen Kirche.

\section{Anmerkungen:}

1. Prakke, Henk/Stoll, Gerhard E.: Entwurf zu einer Religionspublizistik. Communicatio Socialis 1 (1968), S. 35.

2. Dröge, Franz W./Lerg, Winfried B.: Kritik der Kommunikationswissenschaft. Publizistik 10 (1965), S. 262.

3. Rühl, Manfred: Systemdenken und Kommunikationswissenschaft. Publizistik 14 (1969), S. 192.

4. Zum Funktionsbegriff vgl. Luhmann, Niklas: Funktion und Kausalität. Kölner Zeitschrift für Soziologie und Sozialpsychologie 14 (1962), S. 623.

5. Broom, Leonard/Selznick, Philip: Sociology. A Text with Adapted Readings. 4. Aufl. New York 1968, S. 326.

6. Wach, Joachim: Religionssoziologie. Tübingen 1951, S. 172; Janowsky, Bernd: Bürokratie, in: Grochla, Erwin (Hrsg.): Handwörterbuch der Organisation. Stuttgart 1969, Sp. 327; König, René: Bürokratisierung, in: ders. (Hrsg.): Soziologie. Frankfurt/Main 1958, S. 46 f/S. 52. 
7. Etzioni, Amitai: Soziologie der Organisationen. München 1967, S. 95 ff/S. 103 f/S. 112; ders.: Organizational Control Structure, in: March, James G. (Hrsg.): Handbook of Organizations. Chicago 1965, S. 665 f; Eichmann, Eduard/Mörsdorf, Klaus: Lehrbuch des Kirchenrechts auf Grund des Codex Iuris Canonici. 6. Aufl. Paderborn 1949, Band 1, S. 237; Semmelroth, Otto: Amt und Person des Priesters. Stimmen der Zeit 83 (1957/58) Bd. 161, S. 246.

8. Wolf, Ernst: Kirche, in: Beckerath, Erwin von/ . . . Wiese, Leopold von (Hrsg.): Handwörterbuch der Sozialwissenschaften. Stuttgart-Tübingen-Göttingen 1956, Bd. 5, S. 626: "Als societas perfecta ist die Kirche in erster Linie Herrschaftsverband, charakterisiert durch das Gegenüber von lehrender und lernender Kirche, Klerus und Laien, den Begriff des kirchlichen Untertanen (subditus), besondere Formen der Gehorsamsforderung (kirchliche Gebote) und der Jurisdiktion (Kirchenstrafen). ${ }^{*}$

9. Höffner, Joseph: Christliche Gesellschaftslehre. Kevelaer 1962, S. 47: „Staatsverfassung und Staatsform unterliegen dem geschichtlichen Wandel; für die Kirche jedoch ist durch göttliches Recht die hierarchische Verfassung festgelegt. " Vgl. ebenda, S. 231.

10. Etzioni, Amitai: Soziologie der Organisationen, a.a.O., S. 113 f; Banning, W.: Kirche, in: Bernsdorf, Wilhelm/Bülow, Friedrich (Hrsg.): Wörterbuch der Soziologie. Stuttgart 1955, S. 254; Beckel, Albrecht: Subsidiaritätsprinzip, in: Klose, Alfred (Hrsg.): Katholisches Soziallexikon. Innsbruck 1964, Sp. 1207; Messner, Johannes: Kulturethik, in: Klose, Alfred (Hrsg.): a.a.O., Sp. 590.

11. Moberg, David O.: The Church as a Social Institution. Englewood Cliffs. N. J.1962, S. 501; Greinacher, Norbert: Amt ohne Berufsbild, in: ders./Risse, Heinz Theo (Hrsg.): Bilanz des deutschen Katholizismus. Mainz 1966, S. 70 f; Eichmann, Eduard/Mörsdorf, Klaus: a.a.O., S. 236; Höffner, Joseph: a.a.O., S. 232.

12. Külp, Bernhard: Autorität, in: Klose, Alfred (Hrsg.): a.a.O., Sp. 65 ff; Hirsch, Ernst: Moral (Sittlichkeit, Form der), in: Bernsdorf, Wilhelm/Bülow, Friedrich (Hrsg.): a.a.O., S. 340 f; Wach, Joachim: a.a.O., S. 173; Troeltsch, Ernst: The Emergence of Types of Religious Organization, in: Merton, Robert K./Gray, Ailsa P./Hodkey, Barbara/Selvin, Hanan C. (Hrsg.): Reader in Bureaucracy. 2. Aufl. Glencoe Ill. 1960, S. 83/S. 274; Eichmann, Eduard/Mörsdorf, Klaus: a.a.O., S. 235 f; Golomb, Egon: Auch die Kirche muß ihren Einsatz planen, in: Greinacher, Norbert/Risse, Heinz Theo (Hrsg.): a.a.O., S. 47; May, Georg: Kirchenorganisation, in: Grochla, Erwin (Hrsg.): a.a.O., Sp. 807.

13. Greinacher, Norbert: Auf dem Wege zur Gemeindekirche, in: ders./Risse, Heinz Theo (Hrsg.): a.a.O., S. $18 \mathrm{ff}$.

14. Kortzfleisch, Siegfried von: Verkündigung und „öffentliche Meinungsbildung ${ }^{\alpha}$. Stuttgart 1960, S. 187; Schurr, Viktor: Seelsorge in einer neuen Welt. Salzburg 1957, S. 235 f; Reuver, Marco: Publizistischer Arbeitskreis beim Weltlaienkongreß. Communicatio Socialis 1 (1968), S. 46 f; Kraemer, Konrad W.: Resolutionen, in: ders. (Hrsg.): Welt Kirche - Presse. Osnabrück 1969, S. 198 f.

15. Thayer, Lee: Communication and Organization Theory, in: Dance, Frank E. X. (Hrsg.): Human Communication Theory. New York 1967, S. 92 f; Guetzkow, Harold: Communications in Organizations, in: March, James G. (Hrsg.): a.a.O., S. 568.

16. Guetzkow, Harold: a.a.O., S. $542 \mathrm{ff}$; Katz, Daniel/Kahn, Robert L.: The Social Psychology of Organizations. New York 1966, S. 244.

17. Katz, Daniel/Kahn, Robert L.: a.a.O., S. 245 f; Berelson, Bernard/Steiner, Gary A.: Human Behavior. An Inventory of Scientific Findings. New York 1964, S. 370; Simon, Herbert A.: Administrative Behavior. A Study of Decision-Making Processes in Administrative Organization. 2. Aufl. New York 1957, S. 158.

18. Thayer, Lee: Communication and Communication Systems in Organization, Management, and Interpersonal Relations. Homewood Ill. 1968, S. 11/S. $18 \mathrm{ff} / \mathrm{S}$. $98 \mathrm{ff}$; Presthus, Robert V.: Individuum und Organisation. Hamburg 1966, S. 43; Thompson, Victor A.: Hierarchy, Specialization, and Organizational Conflict. Administrative Science Quarterly 5 (1960/61), S. 487 f; Thompson, James D./McEwen, William J.: Organizational Goals and Environment: Goal Setting as an Interaction Process. American Sociological Review 23 (1958), S. 25 ff.

19. Luhmann, Niklas: Funktionen und Folgen formaler Organisation. Berlin 1964, S. 200; Mayntz, Renate: Soziologie der Organisation. Reinbek 1963, S. 95; Ziegler, Rolf: Kommunikationsstruktur und Leistung sozialer Systeme. Mesenheim am Glan 1968, 
S. 118; Litterer, Joseph A.: The Analysis of Organizations. New York 1965, S. 256/ S. 271; Cartwright, Dorwin: The Potential Contribution of Graph Theory to Organization Theory, in: Haire, Mason (Hrsg.): Modern Organization Theory. New YorkLondon 1959, S. 261.

20. Luhmann, Niklas: a.a.O., S. 210; Cartwright, Dorwin: a.a.O., S. 261; Berelson, Bernard/ Steiner, Gary A.: a.a.O., S. 372.

21. Gore, William J./Dyson, J. W.: Introduction, in: dies. (Hrsg.): The Making of Decisions: A Reader in Administrative Behavior. London 1964, S. 1/S. 5; Mayntz, Renate: a.a.O., S. 90; Deutsch, Karl W.: On Communication Models in the Social Sciences. Public Opinion Quarterly 16 (1952), S. 363; Prakke, Henk: Kommunikation der Gesellschaft. Münster 1968, S. 156.

22. Luhmann, Niklas: Soziologie als Theorie sozialer Systeme. Kölner Zeitschrift für Soziologie und Sozialpsychologie 19 (1967), S. 633 f.

23. Voos, Henry: Organizational Communication: A Bibliography. New Brunswick. N. J. 1967, S. $8 \mathrm{f}$; Robinson, James A.: Decision Making in the House Rules Committee, in: Gore, William J./Dyson, J. W. (Hrsg.): a.a.O., S. 320 f; Shull, Fremont/Del Beque, Andre: Norms, A Feature of Symbolic Culture: A Major Linkage Between the Individual, Small Group, and Administrative Organization, in: Gore, William J./Dyson, J. W. (Hrsg.): a.a.O., S. 263; Etzioni, Amitai: a.a.O., S. 122; Gouldner, Alvin: Organizational Analysis, in: Merton, Robert K./Broom, Leonard/Cottrell, Leonard S. Jr. (Hrsg.): Sociology Today. 3. Aufl. New York 1960, S. 404 f.

24. Ziegler, Rolf: a.a.O., S. 202; Hartmann, Heinz: Funktionale Autorität. Stuttgart 1964, S. 113.

25. Hartmann, Heinz: a.a.O., S. 60 f: „Die Gegensätze sind scharf pointiert: Während die Glaubensherrschaft normativ eine Vertiefung und Ausdehnung des Glaubens oder zumindest doch kritiklose Folgeleistung (,Gehorsam') verlangt, besteht unter der funktionalen Autorität ein institutionell verankerter Anreiz zur Herausforderung des Legitimitätsanspruches." Vgl. ebenda, S. 8 ff.

26. Ebenda, S. 61/S. $102 \mathrm{ff}$.

27. Ziegler, Rolf: a.a.O., S. 91; Scott, William G.: Communication and Centralization of Organization. Journal of Communication 13 (1963), S. $3 \mathrm{f}$; Berelson, Bernard/Steiner Gary A.: a.a.O., S. 369; Lerg, Winfried B.: Das Gespräch. Theorie und Praxis der unvermittelten Kommunikation. Düsseldorf 1970, S. $259 \mathrm{f}$.

28. Hartmann, Heinz: a.a.O., S. $43 \mathrm{ff}$; Luhmann, Niklas: Funktionen und Folgen ..., a.a.O., S. 204.

29. Hartmann, Heinz: a.a.O., S. 114 f.

30. Ziegler, Rolf: a.a.O., S. 61.

31. Ebenda, S. 97 f/S. 208 f; Luhmann, Niklas: a.a.O., S. 199; Shaw, Marvin E.: Communication Networks, in: Berkowitz, Leonard (Hrsg.): Advances in Experimental Social Psychology. Bd. 1, New York - London 1964, S. 123.

32. Thompson, Victor A.: Bureaucracy and Innovation. Administrative Science Quarterly 10 (1965), S. $10 \mathrm{ff}$.

33. Hartmann, Heinz: a.a.O., S. 120/vgl. S. 119.

34. Spiegel, Yorick: Kirche als bürokratische Organisation. München 1969, S. 66/S. 73.

35. Hartmann, Heinz: a.a.O., S. 120 f; Spiegel, Yorick: a.a.O., S. 37.

36. Luhmann, Niklas: a.a.O., S. 240.

37. Ebenda, S. 248.

38. Prakke, Henk: a.a.O., S. 108/S. 139 f/S. 157.

39. Molina, P. Antonio: Informations- und Meinungsfreiheit, in: Kraemer, Konrad (Hrsg.): a.a.O., S. 132.

40. Mayntz, Renate: a.a.O., S. 97 f; Spiegel, Yorids: a.a.O., S. 26 f; Greinacher, Norbert: Wege der Wandlung zu einer brüderlichen und kollegialen Kirche. Lebendige Seelsorge 19 (1968), S. 175.

41. Dröge, Franz W.: Publizistik und Vorurteil. Münster 1967, S. $37 \mathrm{ff} / \mathrm{S}$. $61 \mathrm{ff}$.

42. Spiegel, Yorick: a.a.O., S. 40; Vernon, Glenn M.: Sociology of Religion. New York 1962, S. 191. 
43. Boyd, Malcolm: Crisis in Communication. A Christian Examination of the Mass Media. New York 1957, S. 77; Moberg, David O.: a.a.O., S. 489; Dirks, Walter: Fragen an die Kirche, in: Schultz, Hans Jürgen (Hrsg.): Kritik an der Kirche. Stuttgart 1958, S. $136 \mathrm{ff}$; Schreuder, Osmund: Soziologische Aspekte der Verkündigung. Concilium 4 (1968), S. 174; Wach, Joachim: a.a.O., S. 419; Jammes, Jean M.: The Social Role of the Priest. The American Catholic Sociological Review 16 (1955), S. 97.

44. Greinacher, Norbert: Der Glaube wird anders, in: Harenberg, Werner (Hrsg.): Was glauben die Deutschen? München 1968, S. 124: „Das Phänomen, daß rund die Hälfte der Katholiken sich nicht mehr ohne weiteres mit zentralen Wahrheiten des christlichen Glaubens in der bisher tradierten Form identifizieren, deutet doch auf einen grundlegenden Wandel im Verständnis des Glaubens und auf ein verändertes Verhältnis zum Lehramt der Kirche hin." Vgl. Prakke, Henk: Hierarchie der Medien. Zur Diagnose von Kommunikationssystemen. Publizistik 11 (1966), S. 388 f.

45. Greinacher, Norbert (Hrsg.): Priesternachwuchs und Klerus im Bistum Essen. Pastoralsoziologisches Institut des Erzbistums Paderborn und des Bistums Essen, Bericht Nr. 6 (1960), S. $5 \mathrm{f}$; ders. (Hrsg.): Priesternachwuchs und Klerus im Erzbistum Paderborn, a.a.O., Bericht Nr. 10, S. 5 ff/S. 24; ders.: Amt ohne Berufsbild, a.a.O., S. 73; Groner, Franz: Statistik der katholischen Kirchengemeinden in Deutschland, in: Goldschmidt, Dietrich/Greiner, Franz/Schelsky, Helmut (Hrsg.): Soziologie der Kirchengemeinde. Stuttgart 1960, S. 203 f; Harenberg, Werner (Hrsg.): a.a.O., Golomb, Egon: Wie kirchlich ist der Glaube? in: Harenberg, Werner (Hrsg.): a.a.O., S. 174.

46. Paffenholz, Alfred: Katholische Filmbewertung in der Diskussion. Communicatio Socialis 2 (1969), S. $8 \mathrm{f}$.

47. Greinacher, Norbert: Der Glaube wird anders, a.a.O., S. 149: „Das gewandelte Glaubensverständnis der Mitglieder verlangt nach Strukturveränderungen, nach einer neuen kirchlichen Sozialform. "Vgl. ebenda, S. $134 \mathrm{f} / \mathrm{S}$. $142 \mathrm{ff}$.

48. Golomb, Egon: Kollegialität als Strukturprinzip der Kirche. Lebendige Seelsorge 19 (1968), S. 161.

49. Weber, Wilhelm: Soziale Statussymbole in der Kirche im Wandel der Zeit, in: Heinen, Wilhelm (Hrsg.): Bild - Wort -Symbol in der Theologie. Würzburg 1969, S. 268; Spiegel, Yorick: a.a.O., S. 18.

50. Schriftleitung Lebendige Seelsorge: Seelsorge nach dem Konzil. Ein erster Durchblidk durch die Konzilsdokumente. Lebendige Seelsorge 17 (1966), S. 99: „Dem Zentralismus der Kirche wird ....e ein Prinzip der Dezentralisation zur Seite gestellt. Die ,Kollegialität $t^{*}$ ist aber nicht nur ,Korrektiv' gegen einen übersteigerten Zentralismus der Römischen Kurie. Sie ist durchgängiges ,Aufbauprinzip in der Kirche, das auch für die Diözesen und Ortsgemeinden gilt."

51. Golomb, Egon: a.a.O., S. $157 \mathrm{f}$.

52. Ebenda, S. $158 \mathrm{ff}$; Griesl, Gottfried: Krisen im modernen Priesterleben. Lebendige Seelsorge 17 (1966), S. $135 \mathrm{ff}$.

53. Hoefnagels, Harry: Die Krise der kirchlichen Autorität. Die Notwendigkeit einer Demokratisierung der Kirche. Stimmen der Zeit 93 (1968) Bd. 182, S. 149 ff; Golomb, Egon: a.a.O., S. 161; Dreher, Bruno: Theologische Aspekte einer diözesanen Pastoral. Lebendige Seelsorge 16 (1965), S. 117.

54. Hoefnagels, Harry: a.a.O., S. 153; Golomb, Egon: a.a.O., S. 162; Spiegel, Yorick: a.a.O., S. 66 .

55. Hoefnagels, Harry: a.a.O., S. 147/S. 151/S. 155 f; Greinacher, Norbert: a.a.O., S. 133 f/ S. 141.

56. Homeyer, Josef/Stammkötter, Wilhelm/u.a.: Uberlegungen und Vorschläge zur Struktur der Seelsorge im Bistum Münster - Strukturplan. Münster 1969, S. 33/vgl. S. 28 ff.

57. Baukloh, Friedhelm: Für und wider das Bistumsblatt, in: Greinacher, Norbert/Risse, Heinz Theo (Hrsg.): a.a.O., S. $230 \mathrm{ff}$.

58. Spiegel, Yorick: a.a.O., S. $60 \mathrm{f}$.

59. Thayer, Lee: a.a.O., S. 20; Rühl, Manfred: Die Zeitungsredaktion als organisiertes soziales System. Bielefeld 1969, S. 110; Hanssler, Bernhard: Kirche, in: Klose, Alfred (Hrsg.): a.a.O., Sp. 518; Hagemann, Walter: Zur Praxis christlicher Publizistik, in: Jansen-Cron, Heinrich/Bringmann, Karl (Hrsg.): Katholisches Leben und publizistische 
Verantwortung. Heidelberg 1949, S. 114; Hoefnagels, Harry: a.a.O., S. 154; Bahr, Hans Eckehard: Verkündigung als Information. Hamburg 1968, S. 114.

60. König, Franz: Kirche und Kommunikation. Communicatio Socialis 1 (1968), S. 17.

61. Ebenda, S. $13 \mathrm{f}$; Reuver, Mark: Informationsaustausch in religiösen Instituten. Communicatio Socialis 1 (1968), S. 209; Saxer, Ulrich: Massenkommunikation als Mittel christlicher Verkündigung. Communicatio Socialis 2 (1969), S. 112.

62. König, Franz: a.a.O., S. 14; Reuver, Mark: a.a.O., S. 209 f.

63. Bühler, Karl-Werner: Die Kirchen und die Massenmedien. Hamburg 1968, S. $13 /$ vgl. S. 12; Golomb, Egon: Massenkommunikationsmittel, in: Klose, Alfred (Hrsg.): a.a.O., Sp. 665; Heimann, Paul: Film, in: Bernsdorf, Wilhelm/Bülow, Friedrich (Hrsg.): a.a.O., S. 133; Heuvel, Albert van den: Überlegungen zum Thema: Theologie, Kommunikation und Massenmedien. Medium 4 (1967), S.167; Goddijn, Walter/Goddijn, H. P. M.: Kirche als Institution. Einführung in die Religionssoziologie. Mainz 1963, S. 109 f.

\section{S UM M A R Y}

Starting Point: The following text outlines the basic approaches used in a CommunicatorAnalysis of the Catholic clergy on the basis of publicistic organizational sociology. The aim of the analysis is altering of the social status quo of the ecclesiastical hierarchy.

Findings: The Catholic clergy is a highly centralized bureaucratical organization with all of the communications barriers typical for such a group. The clergy alone makes decisions for the Church, which is recognized to be a dominated group. Consequently clerical publicistics is typically a "commando" type of publicistic, which is becoming ever more problematical, indicative of an increasing split between clerical and lay expectations.

Goal: This present conflict or critical situation in the Catholic Church can be solved by her renewing herself and becoming a decentralized and democratic ("collegial") organization. The principal criteria for such a change in the clerical and ecclesiastical communications and decision-making structures are the institutionalizing of a communicative transparency, the adoption of expert knowledge as a legitimization of social influences (in place of "holiness of tradition"), the introduction of decision-making at the very "basis" of the Church, and a permanent process for learning new ways of conducting communications within the Church and society.

\section{RES U MEN}

Planteamiento: El presente esquema circunscribe los elementos fundamentales de una Kommunikator-Analyse (una Sociología Periodística de la Organización) del clero católico. Meta del análisis es la superación del "status quo" social de la Jerarquía eclesiástica.

Dictamen: El clero católico es una organización burocrática centralizada en alto grado, con todas las barreras en la comunicación típicas de tales organizaciones. Ella es la únice que decide sobre una Iglesia que se presenta como organización de poder. Por lo tanto, el periodismo clerical es un ejemplo típico de periodismo "dirigido" que, en vista del derrumbamiento de las expectativas clericales y laicas, cada día es más problemática.

Meta: Esta actual situación de conflicto, respectivamente de crisis, de la Iglesia Católica puede ser reemplazada, mediante la correspondiente innovación, por una organización


ción de las estructuras de decisión y comunicación clericales y eclesiásticas son: institucionalización de la transparencia comunicativa, substitución de la legitimación de la influencia social por la objetividad (en lugar de la "santidad de la Tradición“), formación de la facultad de decisión de la "base ${ }^{\alpha}$ y un permanente proceso de aprendizaje para un nuevo hacer periodístico en la Iglesia y sociedad. 